\title{
Crystal structure control in Au-free self-seeded InSb wire growth
}

\author{
Bernhard Mandl ${ }^{1,2}$, Kimberly A Dick ${ }^{1,3}$, Dominik Kriegner ${ }^{2}$, \\ Mario Keplinger ${ }^{2}$, Günther Bauer ${ }^{2}$, Julian Stangl ${ }^{2}$ and Knut \\ Deppert $^{1}$
}

E-mail: Bernhard.Mandl@jku.at

${ }^{1}$ Department of Solid State Physics, Lund University, S-22 100 Lund, Sweden

${ }^{2}$ Institute of Solid State- and Semiconductor Physics, Johannes Kepler University Linz, A-4040 Linz, Austria

${ }^{3}$ Polymer and Materials Chemistry, Lund University, S-22 100 Lund, Sweden

\begin{abstract}
In this work we demonstrate experimentally the dependence of InSb crystal structure on the ratio of $\mathrm{Sb}$ to In atoms at the growth front. Epitaxial InSb wires are grown by a self-seeded particle assisted growth technique on several different III-V substrates. Detailed investigations of growth parameters and post-growth energy dispersive x-ray spectroscopy indicate that the seed particles initially consist of In and incorporate up to 20 atomic \% Sb during growth. By applying this technique we demonstrate the formation of zinc blende, $4 \mathrm{H}$ and wurtzite structure in the InSb wires (identified by transmission electron microscopy and synchrotron x-ray diffraction), and correlate this sequential change in crystal structure to the increasing $\mathrm{Sb} / \mathrm{In}$ ratio at the particle-wire interface. The low ionicity of $\mathrm{InSb}$ and the large diameter of the wire structures studied in this work are entirely outside the parameters for which polytype formation is predicted by current models of particle seeded wire growth, suggesting that the $\mathrm{V} / \mathrm{III}$ ratio at the interface determines crystal structure in a manner well beyond current understanding. These results therefore provide important insight into the relationship between the particle composition and the crystal structure, and demonstrate the potential to selectively tune the crystal structure in other III-V compound materials as well.
\end{abstract}

PACS numbers: 81.07.Gf,61.46.Km,81.05.Ea,81.15.Gh,62.23.Hj

Submitted to: Nanotechnology 


\section{Introduction}

Semiconductor nanowires represent promising candidates for electronic, optoelectronic, thermoelectric and sensor applications. III-V compound semiconductors exhibit several important properties, among them high electron mobilities, especially for those compounds with narrow direct band gaps. The possibility to grow rather defect-free III-V nanowires epitaxially on substrates with high lattice mismatch, in particular also on $\mathrm{Si}$, opens intriguing avenues for hetero-integration of devices. So far, growth of several III-V compounds in the form of nanowires has been demonstrated, using either foreign seed particles such as Au, or by exploiting self-nucleated or selective area growth schemes. Extensive work has been presented in controlling the nucleation, diameter, length and crystal structure of III-V compound nanowires, primarily using Au seed particles $[1,2]$. For future integration with $\mathrm{Si}$, however, more investigations of Au-free growth techniques are required.

The epitaxial growth of III-V compound nanowires quite often leads to the occurrence of different crystal structures, regardless of the stable bulk phase. While nitride nanowires, which are characterised by a high ionicity, grow preferentially in the hexagonal wurtzite phase, the arsenides with lower ionicity exhibit remarkable polytypism i.e. both hexagonal wurtzite and cubic zinc blende phases. Several factors which influence these structural changes have been discussed, such as wire diameter [3, 4] growth temperature [5], supersaturation [6], or doping and the ratio between the group V and group III compounds during growth. Uncontrolled polytypism is commonly reported in both $\mathrm{Au}-$ seeded and Au-free growth techniques. Since wurtzite and zinc blende are predicted to have different band structures, uncontrolled structural mixing is clearly undesirable for devices; however controlled tuning of crystal structure may open up new possibilities for advanced applications [7].

For the present study we have chosen InSb, as this material has a very low ionicity [8], and thus no "intrinsic driving force" towards the wurtzite structure exists. So far only few studies on one-dimensional growth of InSb exist. Epitaxial InSb nanowires with controlled morphology have been grown using Au seed particles. These nanowires were reported to exhibit single-crystalline zinc blende structure only $[9,10,11,12]$. InSb nanowire growth without the use of foreign seed particles was reported in $[13,14,15]$. However, in these publications no systematic investigation of the influence of different growth parameters on e.g. the wire length and diameter, or the crystal structure was presented. Furthermore, epitaxial self-seeded InSb and GaSb nanowire growth have not yet been demonstrated.

Here we report on the epitaxial growth of InSb wires on several III-V compound substrates seeded by In-rich liquid particles in which significant Sb is incorporated. We show that the crystal phase of these wires depends critically on the ratio of the $\mathrm{Sb}$ and In content within this particle, with pure zinc blende, pure wurtzite and $4 \mathrm{H}$ structure forming for specific $\mathrm{Sb} / \mathrm{In}$ ranges. Since InSb has a low ionicity and the wires in this study have a relatively large diameter for epitaxial nanowires, the crystal 
structure results cannot be explained by current models. These results thus demonstrate the potential for crystal structure tuning well beyond currently anticipated limits, and provide input for phase control techniques for all III-V nanowire materials.

\section{Results}

For the growth of the InSb wires we adapted a method successfully demonstrated for the growth of InAs nanowires [16], using a metal organic vapour phase epitaxy (MOVPE) system. Several III-V compounds were used as substrate materials, namely InAs, InP, GaAs, and GaP. Before loading the substrates into the reactor a $\approx 13 \AA$ thick $\mathrm{SiO}_{x}(\mathrm{x} \approx 1)$ layer was deposited onto the single crystalline semiconductor substrate by thermal sublimation. The capped substrates were annealed in the growth reactor at a temperature of $580^{\circ} \mathrm{C}$ for 1 min before initiating the growth at a growth temperature of $440^{\circ} \mathrm{C}$ (unless otherwise stated). In a previous work we reported that such a $\mathrm{SiO}_{x}$ layer forms openings when annealed at temperatures higher than $500{ }^{\circ} \mathrm{C}$, those openings serve as mobility barriers for In particles and support self-seeded particle-assisted nanowire growth [17]. Once the growth temperature was reached, the precursors were activated simultaneously. Trimethylindium (TMI) and trimethylantimony (TMSb) were used for In and Sb, respectively. The molar fraction for TMI was set at $5.09 \times 10^{-6}$ and for TMSb was varied from $3.42 \times 10^{-5}$ to $4.51 \times 10^{-5}$ for wire growth. As carrier gas $\mathrm{H}_{2}$ at a flow of $13000 \mathrm{ml} / \mathrm{min}$ was employed. The growth of the InSb wires was stopped by terminating the TMI supply; the samples were cooled under a constant TMSb flow. At a temperature of $300^{\circ} \mathrm{C}$ also the TMSb flow was switched off. After a temperature lower than $200^{\circ} \mathrm{C}$ was reached the samples were unloaded from the reactor.

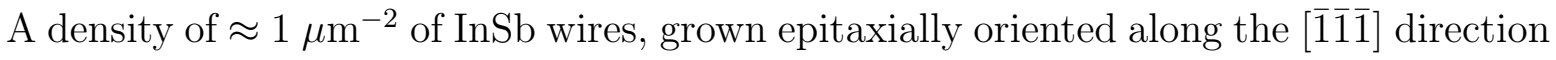
of the substrate, was found on the samples, as shown in the scanning electron microscopy image (Figure 1). In order to determine the epitaxial relationship of the wires with repect to the substrate $\mathrm{x}$-ray diffraction pole figure measurements were performed. As an example a pole figure measurement for InSb wires grown on an InAs (111)B substrate is shown in Figure 2. From these pole figure measurements we can directly obtain the distribution of lattice directions and therefore test if the InSb wires grow epitaxially with respect to the substrat. In Figure 2 the pole figure shows the diffraction spots from InSb $\{111\}$ lattice planes. Epitaxial wires maintaining the substrates inplane orientation were found, e.g. $[11 \overline{2}]_{\text {InSb }}$ parallel $[11 \overline{2}]_{\text {InAs. }}$. The spots indicated by TW result from rotationally twinned parts within the wires. Additional weak signals originating from twins in tilted directions were found too, similarly to previous investgations of $\mathrm{Si}$ and GaAs wires grown on Si substrates [18]. We associate these signals with the InSb

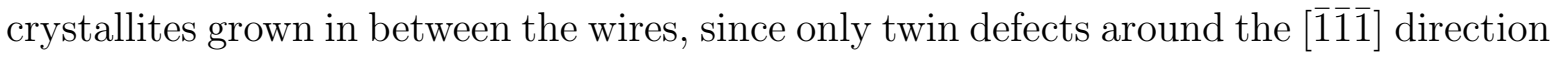
were found by transmission electron microscopy (TEM) investigation of the wires.

The analysis of x-ray diffraction (XRD) experiments allow us also to determine the orientation distribution of the wire lattice plane directions around with respect to the 


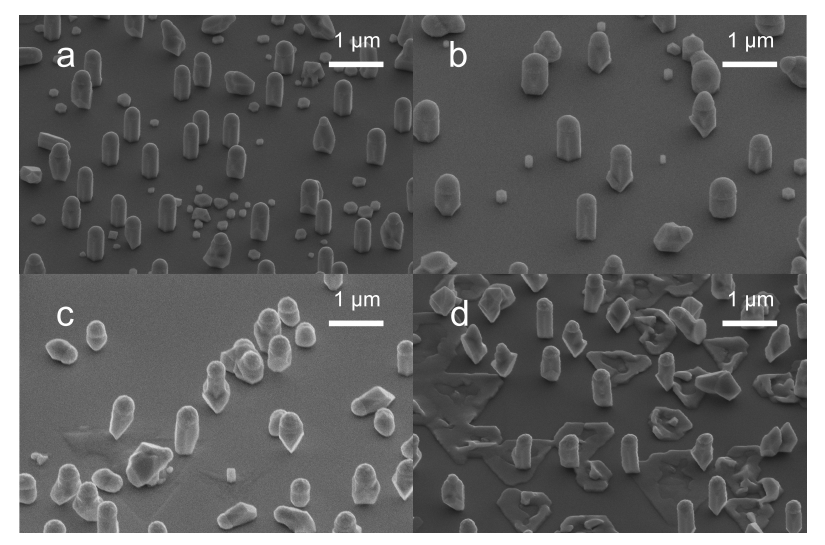

Figure 1. SEM images showing InSb wires on (a) InAs (111)B, (b) InP (111)B, (c) GaAs (111)B and (d) GaP (111)B substrates. MOVPE growth time was 40 min, SEM images were recorded at $45^{\circ}$ sample tilt.

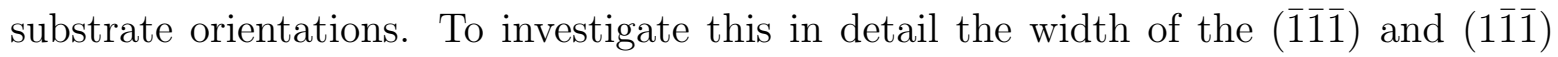
Bragg peaks was measured. The full width at half maximum (FWHM) of the ( $\overline{1} \overline{1} \overline{1})$ Bragg peak in the tilt direction and the FWHM of the (11) 1 ) in the azimuthal direction were found to be 0.37 and 0.45 degree, respectively. As the width of the Bragg peaks of the wires is not only influenced by the orientation distribution but also by the size/thickness of the wires the width of the Bragg peaks represents an upper limit for the width of the orientation distribution.

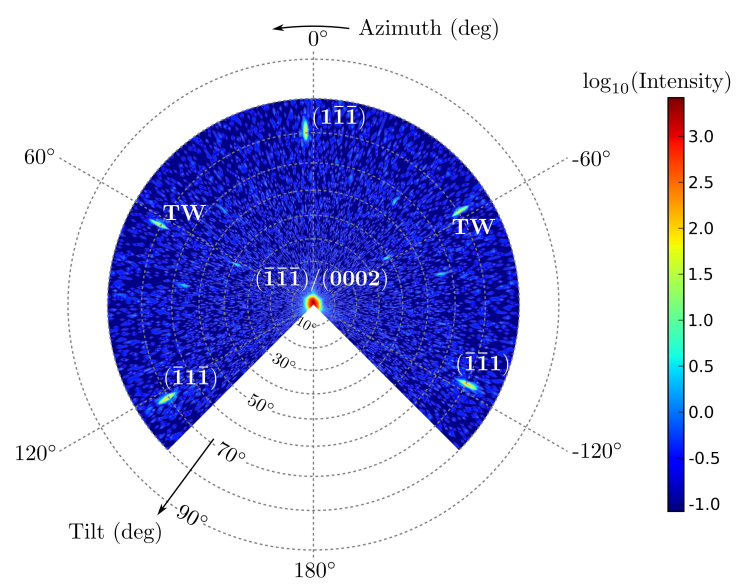

Figure 2. X-ray diffraction pole figure of $\{111\}$ lattice planes of InSb wires grown on InAs (111)B substrate. The pole figure shows the different orientations of the $\{111\}$ lattice planes. Epitaxial growth of the wires with respect to the substrate is found. The rotationally twinned segments within the wires produce additional peaks marked by TW.

The wires have a typical aspect ratio in the range from 2.44 (10 min of growth) to 3.9 (80 min of growth). Thus wires with a length of $\approx 0.9 \mu \mathrm{m}$ exhibit a diameter of 
$\approx 300 \mathrm{~nm}$ (40 min of growth, which is used if nothing else is stated). Figure 1 shows the growth results for the different substrate materials, namely InAs, InP, GaAs, and GaP, all with (111)B surface orientation. The lattice mismatch between these different substrates and InSb ranges from $6.49 \%$ to $15.87 \%$ InSb wire growth is possible on all these substrates regardless of mismatch.

The wire morphology shows three distinct features: (i) for some of the wires at their base, strong faceting is found with facets that are not parallel to the wire growth direction, resulting in a narrower wire diameter at the base, (ii) after a certain wire height, the wires exhibit a hexagonal cross section without any tapering and $\{011\}$ side facets. (iii) a particle is found on top of the wires.

For the characterisation of the role of the seed particle on the wire growth we performed post-growth energy dispersive x-ray spectroscopy (EDXS) on particles at the tips of InSb wires. In all cases the particles consisted primarily of In, but with considerable concentrations of Sb (up to 20 at. \%). However, the linescan analysis of the chemical composition of the particles along the growth direction revealed a significant drop in the $\mathrm{Sb}$ concentration near the interface to the wires, see Figure 3. This decrease seems to correspond to a Sb depletion of the seed particle at the end of growth, which occurs most probably during cooling of the wires down from growth temperature. The existence of this region suggests that the diffusion of Sb through the In at the end of the growth is fairly slow, as such a gradient would otherwise be smoothed out. Therefore, the $\mathrm{Sb}$ concentration away from this interface may be taken as roughly indicative of the $\mathrm{Sb}$ content in the In-Sb seed particle during growth.
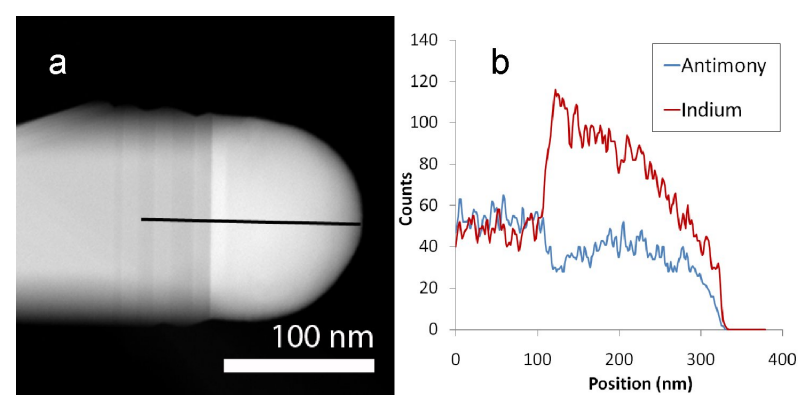

Figure 3. Particle composition at the tip of the InSb wire (a) HAADF-STEM image of the top of an InSb wire (b) EDXS line spectrum of region indicated by the black line in (a). The stoichiometric composition evaluated by quantitative point evaluation of this wire is 50.6:49.3 (In:Sb) with an error of $\pm 1 \%$. In the particle both In and $\mathrm{Sb}$ are found, for details see the text. The particle itself is amorphous. At the interface between wire and particle a depletion of $\mathrm{Sb}$ is found.

In the discussion of the effect of $\mathrm{Sb} / \mathrm{In}$ ratio on wire growth rate and morphology, we distinguish between the nominal $\mathrm{Sb} / \mathrm{In}$ ratio, determined by the molar fractions of $\mathrm{TMSb}$ and TMI precursors, and the effective Sb/In ratio, determined by the concentration of $\mathrm{Sb}$ and In atoms at the growth front. The nominal $\mathrm{Sb} / \mathrm{In}$ ratio was varied by changing the TMSb flow, while the TMI flow was set to a constant molar fraction of $5.09 \times 10^{-6}$. 
No significant effect of the nominal $\mathrm{Sb} / \mathrm{In}$ ratio on either the axial or radial growth rate was observed in the TMSb/TMI range of 6.72 - 8.85. At higher ratios (i.e. for 9.39) no wire growth is observed at all; instead, only crystallites are formed. This suggests that the seed particle either cannot form or cannot be maintained when the Sb flow is too high. The density of wires is also not affected by the nominal V/III ratio in an obvious way. At considerably lower nominal V/III ratios only In droplets are formed on the surface.

Wire growth was performed in the temperature range of $430-450^{\circ} \mathrm{C}$. This range is limited on the one hand due to the relatively high temperature required for cracking of the TMSb precursor $[19,20]$ and on the other hand by the low melting point of InSb $\left(527^{\circ} \mathrm{C}\right)$. No growth is observed at $450^{\circ} \mathrm{C}$. This may be due to a significant increase in the cracking of the TMSb precursor, which leads to an increased effective V/III ratio, consistent with the report above.

Crystal structure characterisation was performed using a JEOL-3000F field emission transmission electron microscope (TEM) operated at $300 \mathrm{kV}$ in conventional TEM mode and in high-angle annular dark field (HAADF)-scanning TEM (STEM) mode. TEM images were recorded along the $\langle\overline{1} 10\rangle$ zone axis (cubic notation) and compositions were determined using EDXS operated in HAADF-STEM mode. In addition to the TEM studies XRD was performed on the InSb wires at beamline D4 at Hasylab Hamburg, where a Rh mirror and a Ge(111) single crystal monochromator were used to provide a monochromatic x-ray beam with an energy of $8944 \mathrm{eV}$.

A TEM analysis of single wires revealed a very interesting structure, see Figure 4, related to the effective $\mathrm{Sb} / \mathrm{In}$ ratio: All wires had zincblende (ZB) structure at their bottom part (100 nm or more), consistent with results previously reported for Au seeded InSb nanowires. However, at a certain distance from the substrate (depending on growth conditions), twin planes appeared in the wires. This was followed by a change to the wurtzite (WZ) crystal structure. The upper section of the longest wires had pure WZ structure without stacking defects. Finally, the very ends of the wires always had a thin $\mathrm{ZB}$ neck region. In addition, most wires exhibit short but significant segments of the $4 \mathrm{H}$ structure between the $\mathrm{ZB}$ and $\mathrm{WZ}$ segments, see Figure $4(\mathrm{c}) .4 \mathrm{H}$ is an intermediate structure between $\mathrm{ZB}$ and WZ, which follows the stacking sequence ABCBABCB... Although this structure has been reported theoretically to be thermodynamically favourable in nanowires, it has so far only been observed experimentally in GaAs nanowires [21].

To confirm the presence of these three crystal structures in our InSb wires, XRD experiments were conducted for the sample grown at a nominal $\mathrm{Sb} / \mathrm{In}$ ratio of 7.7. The different structures can be distinguished in XRD by a crystal truncation rod (CTR) scan at an asymmetric Bragg reflection, where the diffracted Bragg intensity from planes inclined with respect to the $(\overline{1} \overline{1} \overline{1})$ substrate surface is recorded. Scattering from planes parallel to the substrate surface is not sensitive to the different stacking sequences of ZB, $\mathrm{WZ}$, and $4 \mathrm{H}$, because they have very similar lattice plane spacing along $[\overline{1} \overline{1} \overline{1}] /[000 \overline{1}]$ and hence show equal diffraction peak positions. Only the next-next nearest dimer (a pair 


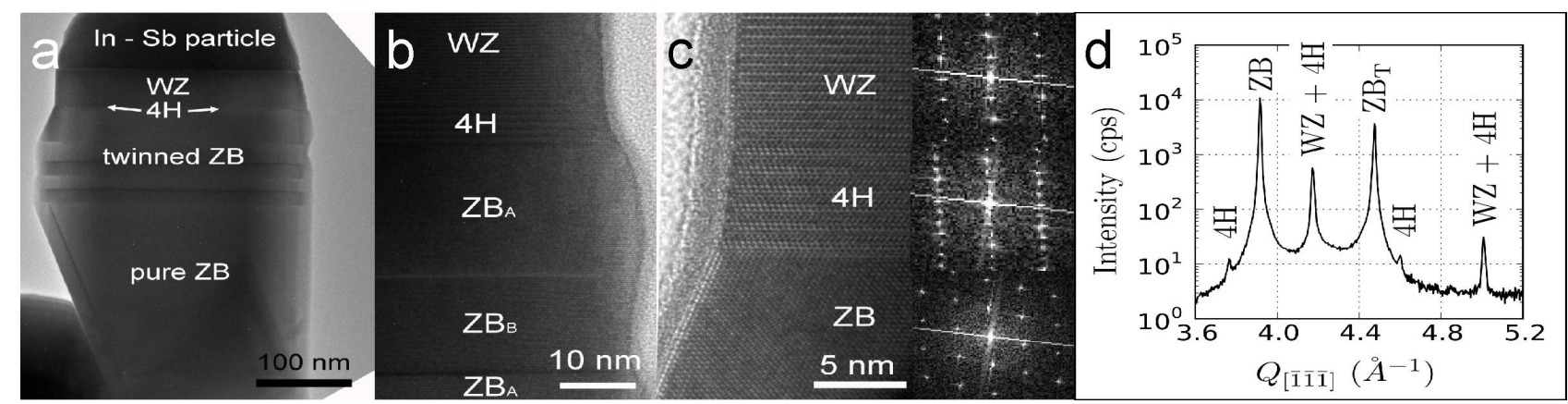

Figure 4. TEM and XRD data of the crystal structure of InSb wires. (a) TEM image along the $<110\rangle$ zone axis of the wire, showing the transition of crystal structure from $\mathrm{ZB}$ to twinned $\mathrm{ZB}$, to $4 \mathrm{H}$ structure and then to WZ. (b) Higher magnification TEM image, with ZB of two orientations (A and B), 4H and WZ. (c) High resolution TEM image showing the $\mathrm{ZB}, 4 \mathrm{H}$ and $\mathrm{WZ}$ crystal structures. Accompanying fast Fourier transforms confirm the 3 different structures (WZ top, $4 \mathrm{H}$ middle and ZB bottom).(d)

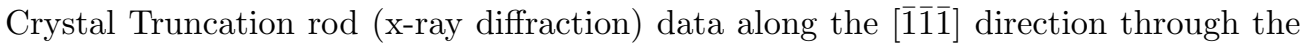
$(\overline{3} \overline{3} \overline{1})$ Bragg peak of InSb. In the scan diffraction signals from $\mathrm{InSb} \mathrm{ZB}, \mathrm{WZ}, 4 \mathrm{H}$ and twinned $\mathrm{ZB}\left(\mathrm{ZB}_{\mathrm{T}}\right)$ is found.

of an In and an Sb atom along the [111] direction) distance varies when the structure is changed, which has shown to only slightly change the lattice spacing [18]. By scanning along a CTR at an asymmetric reflection one is sensitive to the stacking sequences due to the different unit cell sizes the different structures diffract at different positions. In Figure $4(\mathrm{~d})$ such a CTR scan through the InSb $(\overline{3} \overline{3} \overline{1})$ reflection, measured in coplanar scattering geometry, is shown. Peaks associated with the $4 \mathrm{H}$ phase together with more intense peaks which originate from the ZB and WZ phase were observed. The WZ and

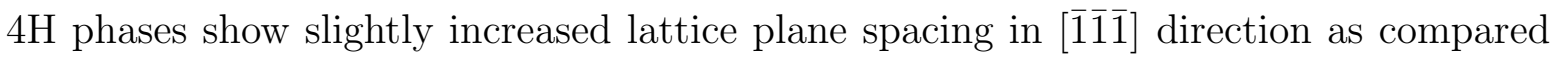
to the ZB phase. The intensity of the peaks in the CTR scan scales with the structure factor and the amount of scattering material. The lower intensity of the $4 \mathrm{H}$ peaks is therefore in agreement with the TEM results, where the $4 \mathrm{H}$ segments were found to be shorter in length than the WZ and ZB segments. In contrast to TEM experiments for which the wires are placed on a lacey carbon $\mathrm{Cu}$-grid by mechanical transfer, the $\mathrm{XRD}$ was performed using as-grown samples, therefore the scattering signal from all structures on the sample is recorded, including surface and island growth as seen in the SEM image of Figure 1.

\section{Discussion}

While the WZ structure is commonly observed in nanowires of many other III-V compounds, and in fact it is common even in materials which do not exhibit WZ structure in their bulk form (such as InAs). For antimonide nanowires the WZ structure has not previously been reported. The appearance of WZ structure was considered to be linked to the high surface area [4], and several theories and models have been 
developed to explain this phenomenon $[3,6]$. However, to date antimonide nanowires (InSb and GaSb) have been the exception among III-V materials, and so far all reports have reported perfect $\mathrm{ZB}$ nanowires free of stacking defects $[9,10,22,11,12]$, except for GaAsSb nanowires [23]. Several explanations have been proposed for the absence of twin planes, stacking faults and WZ phase in previous reports on III-antimonide nanowires. It is often noted that the ionicity of the III-V compounds affects the relative energy of ZB versus WZ stacking: nitride materials have the highest ionicity of III-V materials and exhibit WZ phase in their bulk form, while other III-V compounds, lower in ionicity, exhibit the stable ZB structure as in bulk materials. Antimonides have the lowest ionicity among the III-V compounds and this fact has been related to the absence of WZ segments in antimonide wires. However, this explanation is unsatisfactory, since a closer look at reported ionicity values [8] indicates that the value for InSb is only slightly lower than for GaAs, and GaAs nanowires typically do exhibit stacking defects and often even WZ phase segments.

A further explanation for the absence of the $\mathrm{WZ}$ phase is that the InSb nanowire diameters reported to date are too large to show the change to the WZ phase. It has been demonstrated that the appearance of $\mathrm{WZ}$ phase in nanowires is diameter-dependent, with a cross-over diameter (below which WZ dominates over ZB structure) characteristic for each material [3]. Thermodynamic models indicate that the antimonides should have the smallest cross-over diameter of all III-V compounds, possibly around $10 \mathrm{~nm}$ [5]. However, although nanowires of other III-V compounds have been reported in this diameter range, antimonide nanowires with diameters below $25 \mathrm{~nm}$ have not yet been grown along the $\langle 111\rangle$ directions [10].

According to some models a significant dependence of structure and cross-over diameter on growth conditions should exist, and it has been proposed that typical antimonide nanowire growth conditions do not allow for the WZ structure to be reached. Indeed the temperature window for growth of these materials is quite small, both due to the low decomposition temperatures of InSb and $\mathrm{GaSb}$, and the relatively limited selection of Sb-containing precursor materials. In fact, for typical MOVPE growth conditions there is a very small parameter space where a reasonable supply of Sb can be obtained by thermal cracking of available precursors, while the temperature is still low enough, so that formation of InSb is thermodynamically favoured. It has been suggested that the achievable Sb flux gives too low supersaturation for stacking defects to form (with WZ normally being associated with high supersaturation in nanowire growth [24, 25]). Our observation of stacking defects and WZ phase in InSb wires gives significant insight into the question of why these structures are usually not observed for InSb nanowires. It clearly rules out the suggestion that WZ antimonide materials are "impossible" to grow due to ionicity or other material parameters. As well, the very large diameter of the wires of our samples $(200-300 \mathrm{~nm})$ also rules out any effect of a small cross-over diameter compared to wire growth achieved so far. In fact, the large diameter suggests that appropriate growth conditions should be achievable for nanowires of any diameter. Consequently we suggest as the most probable scenario that the formation of WZ and 
$4 \mathrm{H}$ segments is entirely controlled by growth conditions. We first note that the growth temperature used here, and the metal-organic In and Sb precursor types and flows, are in the same range as for gold-seeded InSb nanowire growth $[9,10]$. This shows that a sufficient $\mathrm{Sb}$ flux for $\mathrm{WZ}$ or $4 \mathrm{H}$ formation can be achieved using these parameters. The only difference between the growth mode reported here and earlier reports is that In-Sb particles are used as seeds rather than gold nanoparticles. This obviously gives a very different effective $\mathrm{Sb} / \mathrm{In}$ ratio and supersaturation of growth materials at the particle-wire interface. An understanding of these parameters should give us insight into the conditions under which stacking defects and WZ phases form in InSb wires and consequently an indication for other III-V compounds.

Therefore, we investigated in detail how the wires evolve with growth time. Wires were grown for times ranging from 5 to 80 minutes in order to determine the effect of growth time on wire length, diameter, density and structure (described below). Both length and diameter increase monotonously with growth time. For a growth duration of 10 min the wires have a diameter of $173 \pm 20 \mathrm{~nm}$ and a length of $421 \pm 50 \mathrm{~nm}$, while for 80 min the diameter increases to $327 \pm 42 \mathrm{~nm}$ and the length to $1272 \pm 59 \mathrm{~nm}$.

EDXS performed on the In-Sb particles at the tops of the wires reveals a temporal dependence of their chemical composition. For wires grown for 40 minutes, an $\mathrm{Sb}$ concentration in the particle of up to 20 at. \% was measured. Wires grown for 20 minutes had a typical maximum Sb composition of 7-8 at. \%, while wires grown for 10 minutes had a maximum Sb composition of only 3-4 at. \%. Examining the crystal structure of these wires as a function of growth time, it is possible to correlate this structure with the Sb composition of the particle. Wires grown for 40 minutes exhibited pure WZ structure at the end, except for the thin ZB neck region typically formed during cooling [26]. Examining the wires grown for 20 minutes, we see that the structure at the end contains numerous stacking defects (and in some cases a $4 \mathrm{H}$ phase); but typically no pure WZ phase is reached. Finally, wires grown for only 10 minutes had pure ZB structure. From that we see that while ZB formation may be associated with a low concentration of $\mathrm{Sb}(3-4$ at. \%) in the In-Sb particle, at intermediate concentrations (7-8 at. \%) a mixed structure forms, and for high Sb concentrations ( $\approx 20$ at. \%) the crystal structure changes to WZ.

For long growth times ( $80 \mathrm{~min}$ ), a shrinking or even the disappearance of the In-Sb particle at the wire tip is observed, see Figure 5. As described above, with increasing growth time the Sb concentration in the seed particle increases, but no steady state seems to be reached. For the In - Sb system the only stable intermediate phase is stoichiometric InSb [27]. Thus it is reasonable to assume that for prolonged growth the $\mathrm{Sb}$ concentration in the particle increases until a concentration very close to $50 \% \mathrm{Sb}$ is reached, which leads to rapid crystallisation and hence "consumption" of the In-Sb particle: As the diffusion of Sb inside the particle is obviously a slow process, the seed particle gradually shrinks. This assumption is supported by the shrinking of diameter for wires grown for $80 \mathrm{~min}$. It is quite reasonable to assume that In and $\mathrm{Sb}$ have different diffusion lengths on the substrate and on the wire side walls. The increase of the Sb 
concentration in the seed particle may indicate that In has a shorter diffusion length and that by increasing the wire length the effective $\mathrm{Sb} / \mathrm{In}$ ratio increases within the particle, leading to the observed effect of its collapse. This fits also very well with the limit for wire growth for too-high nominal Sb/In ratios or too-high temperatures during growth, since both these conditions are expected to be related to a high effective $\mathrm{Sb} / \mathrm{In}$ ratio in the growth particle, leading to crystallisation before wire growth can nucleate.

In principle a change of the temperature at the particle wire interface with increasing wire length could influence nucleation conditions, however for the dimensions in our case no significant temperature drop is to be expected [28].

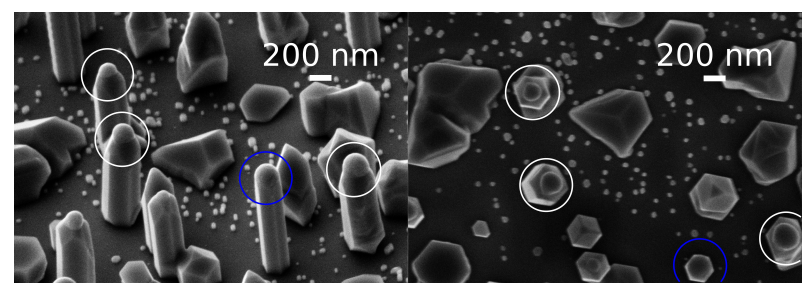

Figure 5. SEM images showing InSb wires on InAs (111)B substrates after MOVPE growth of $80 \mathrm{~min}$. Left: Substrate is tilted by $45^{\circ}$. In the white circles, wires with "collapsing particles" are marked. A conical shape at the top can be seen, with a small particle at its end. In the extreme case the cone has a sharp tip and no particle can be found anymore. Untapered wires are found as well (blue circle). Right: The same set of wires is recorded in a top view. It can clearly be seen that all wires exhibit hexagonal cross-section. Furthermore both the particle and the wire diameter shrink, with the particle staying in the center of the wire.

While the determined ex-situ compositions needs not generally be identical to the in-situ compositions, for the reasons mentioned above, the ex-situ composition can certainly be used to compare conditions in the particle during growth (effective $\mathrm{Sb} / \mathrm{In}$ ratio) and relate them to structure. This means that the increasing supersaturation in the In-Sb droplet drives the transition from ZB towards WZ in the growing wire.

Comparing our results to gold-seeded InSb nanowire growth, we find that the precursor flows (molar fractions of $4.08 \times 10^{-5}$ for TMSb and $5.30 \times 10^{-6}$ for TMI, respectively), as well as the ratio between them are within precisely the same range normally used for Au-seeded nanowires [10]. Nevertheless, ex-situ EDXS analysis performed on Au-seeded InSb nanowires typically indicates a composition ration around 32:66:2 for Au:In:Sb [9]. Clearly it is very difficult to compare such compositions, and it is not at all clear how well this correlates to in-situ compositions, as both In and Sb compositions in the seed particle may decrease during cooling due to InSb growth. While from that we can not obtain quantitative numbers, the data suggest that the effective $\mathrm{Sb} / \mathrm{In}$ ratio at the interface may be very low during typical gold-seeded InSb nanowire growth - resulting in pure ZB structure.

This work demonstrates how the nanowire crystal phase can be controlled by the effective $\mathrm{Sb} / \mathrm{In}$ ratio at the particle wire interface far beyond the predictions by current models. It demonstrates the necessity to study this effect also for other III-V nanowire systems 
and furthermore that there is a need for theoretical models describing the influence of the $\mathrm{V} / \mathrm{III}$ ratio on the formation of nanowire crystal structure polytypes.

\section{Conclusions}

In conclusion, we have demonstrated $[\overline{1} \overline{1} \overline{1}]$ or $[000 \overline{1}]$ oriented epitaxial growth of InSb wires using a self-seeded growth technique on several III-V compound substrates. These wires grow using self-assembled In particles, in which a substantial amount of $\mathrm{Sb}$ is dissolved to give a growth mode similar to vapor-liquid-solid phase epitaxy. These antimonide wires exhibit an unusual crystal structure with transitions from pure ZB at the beginning of growth to pure WZ at their end. This observation is most likely correlated with an increase of the Sb composition in the In-Sb seed particle, indicating that crystal structure in antimonide nanowires and most likely in most III-V compounds is strongly determined by the V/III element ratio at the particle-crystal interface. The results allow fundamental insight into the dependence of crystal structure on growth parameters showing that polytype formation in nanowire growth strongly depends on the $\mathrm{V} / \mathrm{III}$ ratio at the growth front.

\section{Acknowledgments}

This work was carried out within the Nanometer Structure Consortium at Lund University (nmC@LU) and was supported by the Swedish Foundation for Strategic Research (SSF), the Swedish Research Council (VR), and the Knut and Alice Wallenberg Foundation, the EU Projects NODE (cont. no. 015783), SANDiE (cont. no. NMP4CT-2004-500101), AMON-RA (cont. no. 214814) and by the FWF Vienna by the SFB project IRON (F2507-N08). The authors thank the staff at beamline D4/Hasylab Hamburg for assistance with the x-ray diffraction experiments.

[1] P. Caroff, K. A. Dick, J. Johansson, M. E. Messing, K. Deppert, and L. Samuelson. Controlled polytypic and twin-plane superlattices in iii-v nanowires. Nat Nano, 4(1):50-55, January 2009.

[2] Kimberly A. Dick. A review of nanowire growth promoted by alloys and non-alloying elements with emphasis on au-assisted III-V nanowires. Progress in Crystal Growth and Characterization of Materials, 54(3-4):138-173, 2008.

[3] J. Johansson, K. A. Dick, P. Caroff, M. E. Messing, J. Bolinsson, K. Deppert, and L. Samuelson. Diameter dependence of the Wurtzite-Zinc blende transition in InAs nanowires. The Journal of Physical Chemistry C, 114(9):3837-3842, 2010.

[4] R. Leitsmann and F. Bechstedt. Surface influence on stability and structure of hexagon-shaped III-V semiconductor nanorods. Journal of Applied Physics, 102(6):063528, 2007.

[5] Toru Akiyama, Kosuke Sano, Kohji Nakamura, and Tomonori Ito. An empirical potential approach to Wurtzite-Zinc-Blende polytypism in group III-V semiconductor nanowires. Japanese Journal of Applied Physics, 45:L275-L278, 2006.

[6] Kimberly A. Dick, Philippe Caroff, Jessica Bolinsson, Maria E. Messing, Jonas Johansson, Knut Deppert, L. Reine Wallenberg, and Lars Samuelson. Control of III-V nanowire crystal structure by growth parameter tuning. Semiconductor Science and Technology, 25(2):024009, 2010.

[7] P. Caroff, J. Bolinsson, and J. Johansson. Crystal phases in III-V nanowires: From random toward engineered polytypism. Selected Topics in Quantum Electronics, IEEE Journal of, $\operatorname{PP}(99): 18$, 2010. 
[8] Tomoki Yamashita, Kosuke Sano, Toru Akiyama, Kohji Nakamura, and Tomonori Ito. Theoretical investigations on the formation of wurtzite segments in group III-V semiconductor nanowires. Applied Surface Science, 254(23):7668-7671, September 2008.

[9] Philippe Caroff, Jakob B. Wagner, Kimberly A. Dick, Henrik A. Nilsson, Mattias Jeppsson, Knut Deppert, Lars Samuelson, L. Reine Wallenberg, and Lars-Erik Wernersson. High-Quality InAs/InSb nanowire heterostructures grown by Metal-Organic Vapor-Phase epitaxy. Small, 4(7):878-882, 2008.

[10] Philippe Caroff, Maria E Messing, B Mattias Borg, Kimberly A Dick, Knut Deppert, and Lars-Erik Wernersson. InSb heterostructure nanowires: MOVPE growth under extreme lattice mismatch. Nanotechnology, 20(49):495606, 2009.

[11] Daniele Ercolani, Francesca Rossi, Ang Li, Stefano Roddaro, Vincenzo Grillo, Giancarlo Salviati, Fabio Beltram, and Lucia Sorba. InAs/InSb nanowire heterostructures grown by chemical beam epitaxy. Nanotechnology, 20(50):505605, 2009.

[12] Hyun D. Park, S.M. Prokes, M.E. Twigg, Yong Ding, and Zhong Lin Wang. Growth of high quality, epitaxial InSb nanowires. Journal of Crystal Growth, 304(2):399-401, June 2007.

[13] Q. L. Ye, T. Yamada, H. Liu, R. Scheffler, N. Mingo, and R. Leverenz. Wurtzite to zinc blende phase transition in GaAs nanowires induced by epitaxial burying. Materials Research Society Symposium Proceedings, 940:P07-05, 2006.

[14] S. Vaddiraju, M. K. Sunkara, A. H. Chin, C. Z. Ning, G. R. Dholakia, and M. Meyyappan. Synthesis of group III antimonide nanowires. The Journal of Physical Chemistry C, 111(20):7339-7347, May 2007.

[15] Chandrashekhar Pendyala, Sreeram Vaddiraju, Jeong H Kim, Jacek Jacinski, Zhiqiang Chen, and Mahendra K Sunkara. Self-nucleation and growth of group III-antimonide nanowires. Semiconductor Science and Technology, 25(2):024014, 2010.

[16] B Mandl, J Stangl, T Martensson, A Mikkelsen, J Eriksson, LS Karlsson, G Bauer, L Samuelson, and W Seifert. Au-free epitaxial growth of InAs nanowires. NANO LETTERS, 6(8):1817-1821, August 2006.

[17] Bernhard Mandl, Julian Stangl, Emelie Hilner, Alexei A. Zakharov, Karla Hillerich, Anil W. Dey, Lars Samuelson, Günther Bauer, Knut Deppert, and Anders Mikkelsen. Growth mechanism of Self-Catalyzed group III-V nanowires. Nano Letters, 10(11):4443-4449, November 2010.

[18] D Kriegner, M Keplinger, J Stangl, A M Andrews, P Klang, B Mandl, T Mårtensson, M Borgström, K Deppert, L Samuelson, G Strasser, and G Bauer. Determination of the wurtzite content and orientation distribution of nanowire ensembles. Materials Research Society Symposium Proceedings, 1206E:1206-M11-39, 2010.

[19] M. J. Cherng, H. R. Jen, C. A. Larsen, G. B. Strigfellow, H. Lundt, and P. C. Taylor. MOVPE growth of GaInAsSb. Journal of Crystal Growth, 77(1-3):408-417, September 1986.

[20] A. Aardvark, N.J. Mason, and P.J. Walker. The growth of antimonides by MOVPE. Progress in Crystal Growth and Characterization of Materials, 35(2-4):207-241, 1997.

[21] Dasa L. Dheeraj, Gilles Patriarche, Hailong Zhou, Thang B. Hoang, Anthonysamy F. Moses, Sondre Grønsberg, Antonius T. J. van Helvoort, Bjørn-Ove Fimland, and Helge Weman. Growth and characterization of wurtzite GaAs nanowires with Defect-Free zinc blende GaAsSb inserts. Nano Letters, 8(12):4459-4463, December 2008.

[22] Mattias Jeppsson, Kimberly A. Dick, Jakob B. Wagner, Philippe Caroff, Knut Deppert, Lars Samuelson, and Lars-Erik Wernersson. GaAs/GaSb nanowire heterostructures grown by MOVPE. Journal of Crystal Growth, 310(18):4115-4121, August 2008.

[23] D L Dheeraj, G Patriarche, L Largeau, H L Zhou, A T J van Helvoort, F Glas, J C Harmand, B O Fimland, and H Weman. Zinc blende GaAsSb nanowires grown by molecular beam epitaxy. Nanotechnology, 19(27):275605, 2008.

[24] Frank Glas, Jean-Christophe Harmand, and Gilles Patriarche. Why does wurtzite form in nanowires of III-V zinc blende semiconductors? Physical Review Letters, 99(14):146101, October 2007. 
[25] Jonas Johansson, Lisa S. Karlsson, Kimberly A. Dick, Jessica Bolinsson, Brent A. Wacaser, Knut Deppert, and Lars Samuelson. Effects of supersaturation on the crystal structure of gold seeded III-V nanowires. Crystal Growth 83 Design, 9(2):766-773, February 2009.

[26] It is probable that the neck region is associated with the depletion region of the particle, and grew during cooling with lower sb supersaturation and/or lower temperature.

[27] Landolt-bornstein database, multiphase systems, binary systems, phase diagrams and thermodynamics, indium (in-x) binary systems [online]. springer verlag gmbh, heidelberg. http://www.springermaterials.com/pdfs/10.1007/10757413_76.pdf (accessed jul 6, 2010).

[28] Frank Glas and Jean-Christophe Harmand. Calculation of the temperature profile in nanowhiskers growing on a hot substrate. Physical Review B, 73(15):155320, April 2006. 